\title{
Effect of borax additive on the dielectric response of polypyrrole
}

\author{
MEHMET KILIÇ*, YAŞAR KARABUL, ZEYNEP GÜVEN ÖZDEMİR, SEDA ERDÖNMEZ, \\ AYŞE EVRIM BULGURCUOĞLU, SERÇO SERKIS YEŞILKAYA, MUSTAFA OKUTAN and \\ ORHAN İÇELLI
}

Department of Physics, Faculty of Arts and Sciences, Yildiz Technical University, 34220 Istanbul, Turkey

*Author for correspondence (mekilic@yildiz.edu.tr)

MS received 3 April 2017; accepted 7 August 2017; published online 29 March 2018

\begin{abstract}
The main aim of this study is to produce added polypyrrole (PPy) borax composites with high dielectric properties for technological applications. For this purpose, PPy-borax composites with different borax concentrations varying from 10 to $50 \mathrm{wt} \%$ have been prepared. To reveal their structural and morphological attributes, the composites have been characterized by Fourier-transform infrared spectroscopy and scanning electron microscopy. The real and imaginary parts of complex dielectric function, the imaginary component of complex electrical modulus and ac conductivity have been investigated at room temperature as a function of frequency in the range $100 \mathrm{~Hz}-15 \mathrm{MHz}$. It has been found that addition of borax increases the dielectric constant of pure PPy. In this respect, PPy-borax composites with the highest dielectric constant at low frequency may be utilized in charge storing devices. On the other hand, the dielectric loss is also very high in low-frequency region for the composites with high borax content. Exploiting this property, the material may also be used in decoupling capacitor applications. The relaxation mechanisms of the samples have also been determined as non-Debye type. The Nyquist curves of the samples have been analysed for calculating the grain and grain boundary resistance and capacitance values. In conclusion, borax has a promising potential to be used as a cheap and effective filler for improving the dielectric properties of PPy polymer.
\end{abstract}

Keywords. Polypyrrole; borax; dielectric properties; Nyquist plot; non-Debye-type relaxation; Cole-Cole equation.

\section{Introduction}

Conducting polymers have become a highly preferred class of electronic materials because of their facile synthesis, good environmental and thermal stabilities and high electrical and optical properties [1]. In particular, conjugated polymers such as polypyrrole (PPy) exhibit conducting or semiconducting properties. These materials combine electrical properties of metal or semiconductors along with the advantages of polymers such as lighter weight, resistance to corrosion, lower cost, etc. Moreover, conducting polymer composites have adjustable electrical properties, less weight, resistance to corrosion, flexibility and processability. Due to these remarkable features, conducting polymer composites have also received increasing interest for wide technological applications, including solar cells, electrochromic devices, sensors and rechargable batteries [2,3]. Therefore, considerably huge number of research focused on developing new polymer composites, and also creating polymer composites containing various fillers and/or reinforcements $[4,5]$.

As a polymeric composite compound, different materials such as polyethylene (PE), polyurethane (PU), poly(maminobenzenesulphonic) acid (PABS) functionalized singlewalled nanotubes (SWNT) (PPy-SWNT-PABS) have been tried for improving both the electrical and mechanical properties of PPy [6-8]. On the other hand, boron compounds have been utilized in various applications for almost 40 years, since these compounds are nontoxic and also have high thermal and mechanical stabilities. The compounds of boron are broadly utilized in polymers, wood and wood products as fire retardants. In particular, some research works have been carried out on the utilization of a combination of borax and boric acid in wood composites and cellulosic materials. Also, boron compounds have been mostly added directly to polymer matrix as a filler [9]. Among boron powder compounds, borax has remarkable properties such as being eco-friendly, nontoxic and having high dielectric and mechanical properties. Especially, as a filler or additive material for a polymeric composite, borax has remarkable properties such as polar-OH groups, which facilitate strong interactions and mechanical stability [10]. Due to its highly crosslinking capability, borax has been used with poly(vinylalcohol) [11] and glucomannan [12]. Kim et al [13] have investigated morphology and rheology of hyaluronate-poly(vinyl alcohol)-borax polymer blends. They reported the formation of H-bonding with hyaluronate aggregates, through PVA-borax crosslinking as a bridge, leading to a network structure. From this point of view, borax has been used as a very-low-cost filler material, which has been extensively used for fire retardant, to control 
and improve the dielectric properties of PPy for electronic applications.

\section{Experimental}

\subsection{Materials}

Pyrrole $\left(\mathrm{C}_{4} \mathrm{H}_{5} \mathrm{~N}\right)$ and anhydrous iron (III) chloride $\left(\mathrm{FeCl}_{3}\right)$ were purchased from Merck (Germany). Chloroform $\left(\mathrm{CHCl}_{3}\right)$ and methanol $\left(\mathrm{CH}_{3} \mathrm{OH}\right)$ were supplied from Sigma Aldrich. For the synthesis of polypyrrole (PPy), $0.02 \mathrm{~mol}$ pyrrole was dissolved in $70 \mathrm{ml}$ chloroform; $0.055 \mathrm{~mol} \mathrm{FeCl}_{3}$, dissolved in $180 \mathrm{ml}$ chloroform, was added into the afore-mentioned solution in a dropwise fashion and then stirred $24 \mathrm{~h}$ at room temperature. After polymerization, the solution was filtered and washed with chloroform and methanol, respectively. The black powder was dried in an oven at $60^{\circ} \mathrm{C}$ for $24 \mathrm{~h}$.

Disodium tetraborate, i.e., borax $\left(\mathrm{Na}_{2} \mathrm{~B}_{4} \mathrm{O}_{7}\right)$ (Product Number: 221732) has also been purchased from Sigma Aldrich.

\subsection{Preparation of composites}

To obtain PPy-borax composites, $\mathrm{Na}_{2} \mathrm{~B}_{4} \mathrm{O}_{7}$ mineral was mechanically added to the related polymer at different weight percentages $(10,25$ and $50 \mathrm{wt} \%)$. To homogenize the mixtures, each blend was ground with an IKA A11 basic analytical mill at the rotational speed of $10000 \mathrm{rpm}$ for 15 min. For dielectric measurements, the homogenous composite mixtures prepared were compressed with a cold press at room temperature. Each pellet was of $13 \pm 0.02 \mathrm{~mm}$ diameter, $0.500 \pm 0.050 \mathrm{~g}$ weight and 1.9-2.2 $\mathrm{mm}$ thickness. Finally, the samples were annealed at $80^{\circ} \mathrm{C}$ for $2 \mathrm{~h}$.

\subsection{Fourier-transform infrared spectroscopy of composites}

Fourier-transform infrared (FT-IR) spectroscopy analysis has been performed on a Perkin Elmer Spectrum 100 one FTIR spectrometer. All spectra of CPs-borax composites have been taken in the $4000-650 \mathrm{~cm}^{-1}$ range, with a resolution of $2 \mathrm{~cm}^{-1}$ after 4 scans. The measurement was also performed in the transmittance mode.

The FT-IR spectra of pure PPy and borax have been presented in figure 1a. The characteristic vibration peaks for pure PPy and borax are in good agreement with scientific literature [10, 14-20] and listed in table 1.

As shown in figure $1 \mathrm{~b}$, the PPy-borax composites display the bands between 3600 and $3200 \mathrm{~cm}^{-1}$, which represent the coordination of $\mathrm{OH}$ groups in borax and hydrogen bonding between $\mathrm{OH}$ groups [21]. The bands at 1695 and $1650 \mathrm{~cm}^{-1}$ are also assigned to the $\mathrm{H}-\mathrm{O}-\mathrm{H}$ bending mode, which shows the compound containing the water of crystallization. The band at $1425 \mathrm{~cm}^{-1}$ belongs to the antisymmetric stretching vibrations of $\mathrm{BO}_{3}$ [20]. In addition, a new band at $1190 \mathrm{~cm}^{-1}$ has been observed for PPy-borax composites. This new band is assigned to the in-plane bending of $\mathrm{B}-\mathrm{O}-\mathrm{H}$ [21]. The band
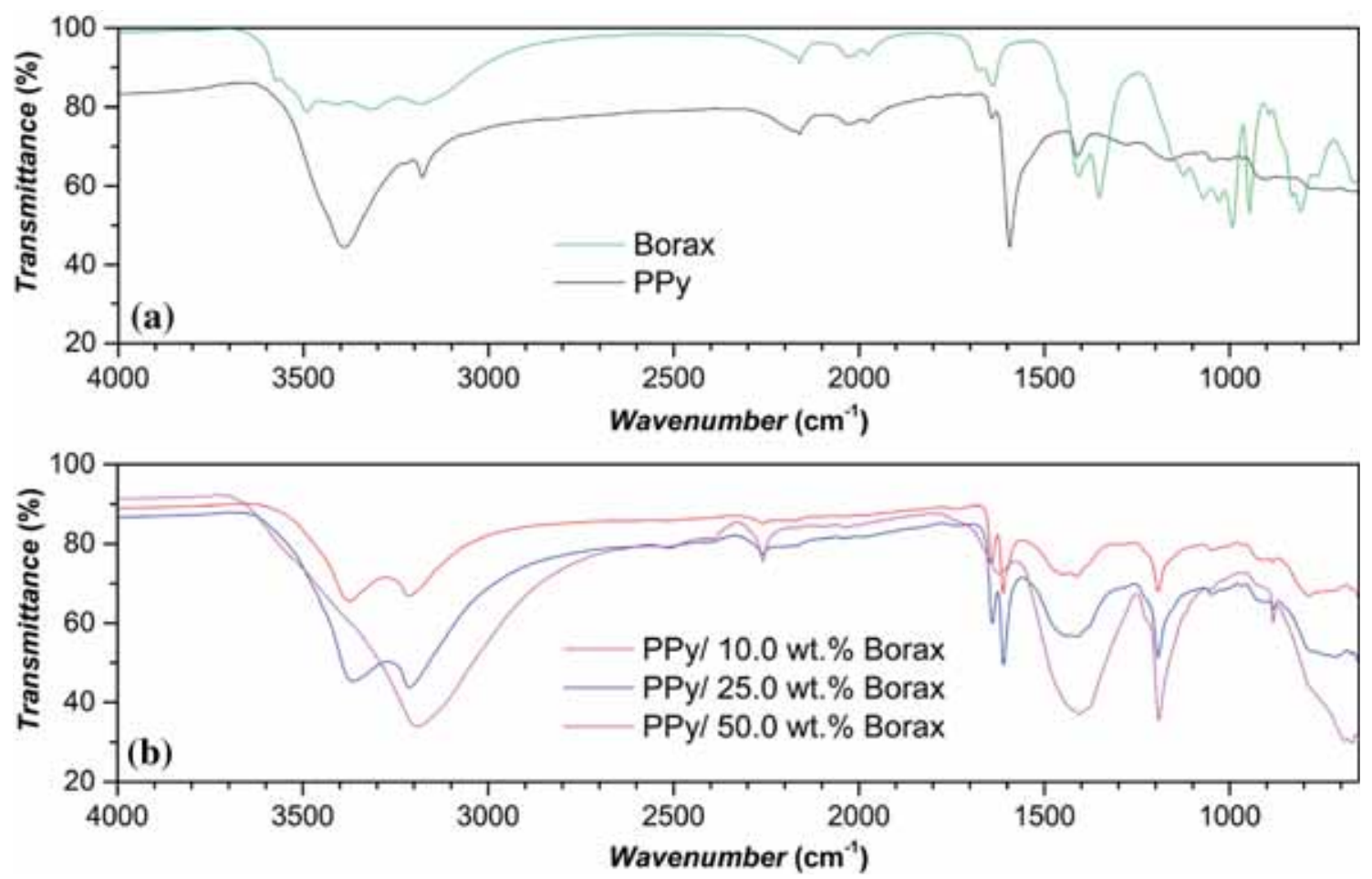

Figure 1. FT-IR spectra of pure PPy and PPy-borax composites. 
Table 1. IR transmittance bands of PPy and borax [10,14-20].

\begin{tabular}{ccc}
\hline & Wavenumber $\left(\mathrm{cm}^{-1}\right)$ & Type of vibration \\
\hline PPy & 781 & $\mathrm{C}-\mathrm{H}$ wagging vibration \\
909 & $\mathrm{C}-\mathrm{H}$ out-of-plane deformation \\
& $\mathrm{C}-\mathrm{H}$ in-plane vibration \\
& 1045 & $\mathrm{C}-\mathrm{N}$ stretching vibration \\
& 1596 & $\mathrm{C}=\mathrm{C} / \mathrm{C}-\mathrm{C}$ stretching vibration \\
3381 & Broad band N-H stretching vibration \\
Borax & BOH in-plane bending vibration \\
& 915,834 & Antisymmetric stretching vibration of tetrahedral $\mathrm{BO}_{4}$ groups \\
& 1130 & B-O bonds in tetrahedral geometry \\
& 1353,1420 & Antisymmetric stretching vibration of $\mathrm{BO}_{3}$ groups \\
3323 & OH stretching vibration \\
\end{tabular}
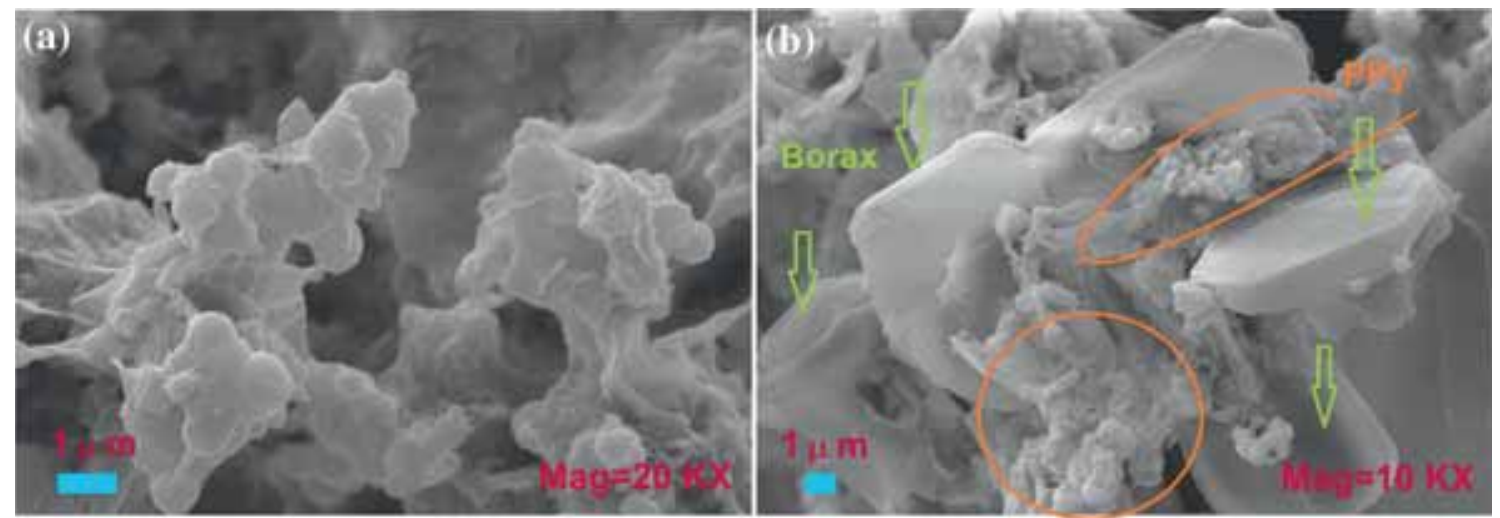

Figure 2. The SEM micrographs of (a) pure PPy and (b) PPy-50 wt\% borax composite.

over $790-670 \mathrm{~cm}^{-1}$ is due to the out-of-plane bending of B-O-H.

\subsection{Scanning electron microscopy of composites}

The surface morphology of the PPy-borax composite samples has been visualized using a Zeiss-EVOR LS 10 scanning electron microscope (SEM). The SEM micrographs for pure PPy and PPy-50 wt\% borax composite have been shown in figure 2 .

It is clear from figure 2 that the micrograph shows a very irregular surface for pure PPy and PPy-borax composite samples. In figure 2a, the surface for pure PPy was enlarged 20000 times. Pure PPy displays a typically cauliflower-like structure. On comparing the SEM images of pure PPy and PPy-borax composite samples, borax heaps in the structure can be easily seen. This means that borax did not diffuse into the PPy and also did not dissolve but borax additive resulted in the formation of a composite structure. Dielectric results, which are presented later, change with borax concentration. Therefore, borax additive is effective on the properties of composite material.

\subsection{Particle size of composites}

The particle size distributions of pure PPy, borax and PPyborax composite have been determined using a Mastersizer, Malvern Hydro 2000MU model VBR and are shown in figure 3 . As seen from figure 3

- size of $90 \%$ of the PPy particles is smaller than 266.243 $\mu \mathrm{m}, 50 \%$ of the PPy particles is smaller than 110.346 $\mu \mathrm{m}$ and $10 \%$ of the PPy particles is smaller than 37.938 $\mu \mathrm{m}$;

- size of $90 \%$ of the borax particles is smaller than $481.982 \mu \mathrm{m}, 50 \%$ of the borax particles is smaller than $115.173 \mu \mathrm{m}$ and $10 \%$ of the borax particles is smaller than $15.185 \mu \mathrm{m}$;

- size of $90 \%$ of the PPy-borax composites' particles is smaller than $122.419 \mu \mathrm{m}, 50 \%$ of the PPy-borax composites particles is smaller than $40.165 \mu \mathrm{m}$ and $10 \%$ of the PPy-borax composites particles is smaller than $10.851 \mu \mathrm{m}$.

According to Mastersizer measurements results, the $50 \mathrm{wt} \%$ borax-doped composite material has lower particle sizes relative to pure PPy and borax. 




Figure 3. Particle size distribution of (a) pure PPy, (b) pure borax and (c) PPy-50 wt\% borax composites.

\section{Results and discussion}

\subsection{Dielectric measurements}

Dielectric measurements have been performed using a HP 4194A Impedance Analyzer in the frequency range between $100 \mathrm{~Hz}$ and $15 \mathrm{MHz}$ at room temperature with a high accuracy $(0.17 \%$ typically). In this work, the overall errors in the dielectric measurements were $2.5 \%$ and the RMS amplitude of the instruments was $\sim 500 \mathrm{mV}$. The samples were placed between two gold electrodes as a sandwich (Au-samples$\mathrm{Au}$ ) whose surfaces wholly overlap the faces of the pellets. The dimension of the electrodes was $25 \times 20 \mathrm{~mm}^{2}$. The active electrode area was $1.327 \times 10^{-4} \mathrm{~m}^{2}$.

\subsection{Complex dielectric and modulus analysis}

The complex dielectric function of the samples has been described by $\varepsilon^{*}(\omega)$ as follows:

$$
\varepsilon^{*}(\omega)=\varepsilon^{\prime}(\omega)-i \varepsilon^{\prime \prime}(\omega)
$$

where $i^{2}=-1 ; \varepsilon^{\prime}(\omega)$ and $\varepsilon^{\prime \prime}(\omega)$ represent the real and imaginary parts of complex dielectric function, respectively. As is known, while the real part of complex dielectric function indicates energy storage ability, the imaginary part of complex dielectric function identifies energy loss. In addition, the real and imaginary components of complex dielectric function depend on each other through the loss tangent $\operatorname{tg} \delta$ as follows:

$$
\varepsilon^{\prime \prime}(\omega)=\varepsilon^{\prime}(\omega) t_{\mathrm{g}} \delta
$$

$\varepsilon^{\prime}=f(\omega)$ and $\varepsilon^{\prime \prime}=f(\omega)$ curves of pure PPy and PPy-borax composites have been presented in figure $4 \mathrm{a}$ and $\mathrm{b}$, respectively. As seen from figure $4 \mathrm{a}$, the real component of complex dielectric function decreases with increasing frequency and then saturates to its lowest value in the high-frequency region. The general dispersive behaviour of $\varepsilon^{\prime}=f(\omega)$ curves in low-frequency region (628 $\mathrm{rad} \mathrm{s}^{-1}<\omega<10^{5} \mathrm{rad} \mathrm{s}^{-1}$ ) can be explained by the fact that the electric dipoles in the materials orient themselves along the electric field applied, which corresponds to a polarization process. However, the nearly constant value of $\varepsilon^{\prime}$ observed at high frequencies can be attributed to the fact that the electric dipoles hardly be able to orient themselves in the direction of the electric field applied above $10^{5} \mathrm{rad} \mathrm{s}^{-1}$. The low- and high-frequency behaviours of the real part of complex dielectric function of the samples can be explained by the bi-layer structure of the dielectric material proposed by Koop [22-24]. According to his model, dielectric material is considered as good conducting grains surrounded by the poorly conducting grain boundaries. From this point of view, charge carriers of the material can move to grain boundaries by hopping or another conducting mechanisms at low frequencies. If the resistance of grain boundary is very high, charge carriers start to accumulate at the grain boundary, resulting in a high-order polarization. The polarization in this case is also called as 'space charge polarization'. The pile-up of electrons at the grain boundary manifests itself as an observation of high $\varepsilon^{\prime}$ values at low frequencies. On the other hand, the probability of electrons reaching the grain boundary decreases at high frequencies, which results in a reduction in polarization. The decrease in polarization at high frequencies is characterized by a behaviour of $\varepsilon^{\prime}$ that is approximately independent of frequency.

On the other hand, while $10 \mathrm{wt} \%$ borax additive slightly decreased $\varepsilon^{\prime}$ values at low frequencies, $50 \mathrm{wt} \%$ borax additive enhanced $\varepsilon^{\prime}$ values by $10^{3}$ times. In this context, the maximum charge storage ability has been achieved for PPy-50 wt $\%$ borax composite. The same behaviour has been observed for polypropylene-graphite composite by Panwar et al [25]. From this point of view, PPy-50 wt\% borax composite may be utilized in charge storing devices.

To investigate the dielectric relaxation mechanism of PPy and PPy-borax composites, $\varepsilon^{\prime}=f(\omega)$ curves have also been fitted by Origin Lab 8.5 graphic program in terms of 

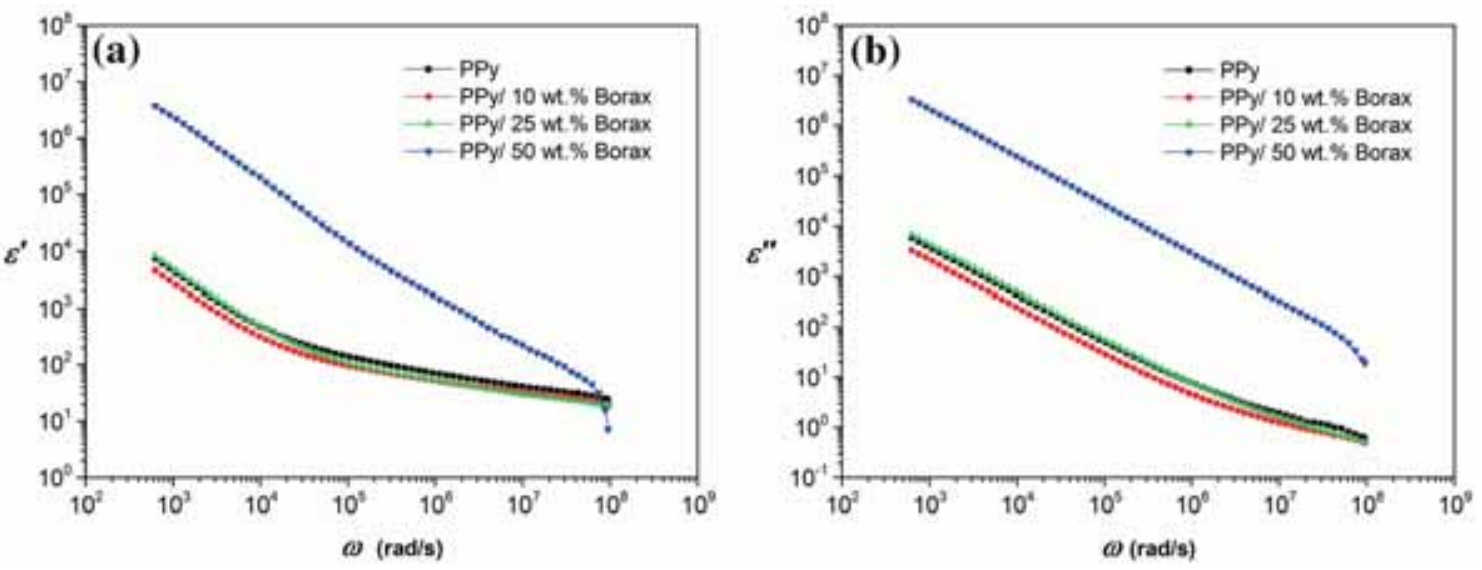

Figure 4. Angular frequency dependence of (a) the real part and (b) the imaginary part of complex dielectric function of pure PPy and PPy-borax composite samples.

Table 2. Absorption coefficient $(\alpha)$, relaxation times $\left(\tau_{0}\right)$, the high and low limiting angular frequency dielectric constants $\left(\varepsilon_{\infty}\right.$ and $\left.\varepsilon_{\mathrm{S}}\right)$ and relaxation strength $(\Delta \varepsilon)$ values for pure PPy and PPy-borax composites.

\begin{tabular}{lcccccc}
\hline Sample & $R^{2}$ & $A$ & $\tau_{0}(\mathrm{~ms})$ & $\varepsilon_{\mathrm{s}}$ & $\varepsilon_{\infty}$ & $\Delta \varepsilon$ \\
\hline PPy & 0.8340 & 0.1542 & 7.21 & 61255.77274 & 55.4305 & 61200.3422 \\
PPy-10 wt\% borax & 0.9867 & 0.1657 & 6.09 & 29992.07482 & 40.9117 & 29951.1630 \\
PPy-25 wt\% borax & 0.9958 & 0.1601 & 4.86 & 43140.45374 & 34.7351 & 43105.7186 \\
PPy-50 wt\% borax & 0.9994 & 0.1584 & 2.70 & $1.14292 \times 10^{7}$ & 11.2460 & $1.1429 \times 10^{7}$ \\
\hline
\end{tabular}

the Cole-Cole equation. The Cole-Cole equation is given as follows:

$$
\begin{aligned}
& \varepsilon^{\prime}(\omega)=\varepsilon_{\infty} \\
& \quad+\left(\varepsilon_{\mathrm{s}}-\varepsilon_{\infty}\right) \frac{1+\left(\omega \tau_{0}\right)^{1-\alpha} \sin \frac{1}{2} \alpha \pi}{1+2\left(\omega \tau_{0}\right)^{1-\alpha} \sin \frac{1}{2} \alpha \pi+\left(\omega \tau_{0}\right)^{2(1-\alpha)}},
\end{aligned}
$$

where $\varepsilon_{\infty}$ and $\varepsilon_{\mathrm{s}}$ are the high and low limiting angular frequency dielectric constants; $\tau_{0}$ and $\alpha$ represent relaxation time and absorption coefficient, respectively [26,27].

The absorption coefficient takes values between zero and one $(0<\alpha \leq 1)$ when adsorption coefficient equals zero, it corresponds to standard Debye-type relaxation. The non-Debye type also occurs when the value of absorption coefficient varies in the $(0,1)$ region [28]. The fitting results in accordance with Cole-Cole equation have been listed in table 2 .

As seen from table 2, absorption coefficient values of all samples are greater than zero. Due to this reason, relaxation mechanism has been considered as non-Debye type for all samples. In addition, the relaxation strength $\Delta \varepsilon$, which is a measure of overall polarizability of the dielectric material, has also been calculated as follows:

$$
\Delta \varepsilon=\varepsilon_{\mathrm{s}}-\varepsilon_{\infty}
$$

According to table 2, while PPy with 10 and $25 \mathrm{wt} \%$ borax added samples have lower relaxation strength values relative to pure PPy, $50 \mathrm{wt} \%$ borax composite has the highest relaxation strength value. The high relaxation strength value observed for $50 \mathrm{wt} \%$ borax additive composite can be associated with occurrence of high population of a very mobile group of dipoles in the sample [29]. In other words, $50 \mathrm{wt} \%$ borax additive makes dipole orientation easier relative to pure PPy. In addition, the decrease in relaxation time with increasing additive concentration also confirms the high population of a very mobile group of electric dipoles in PPy-50 wt\% borax composite.

The angular frequency dependence of $\varepsilon^{\prime \prime}$ also showed a decreasing behaviour in the low-frequency domain and became frequency independent in the high-frequency region (see figure 4b). This general tendency of dielectric loss of all samples can be associated with high and low resistivities in the low- and high-frequency regions, respectively [30]. Moreover, decreasing $\varepsilon^{\prime \prime}$ values with increasing frequency may be associated with high periodic reversal of the field at the interface. As shown in figure $4 \mathrm{~b}$, while $10 \mathrm{wt} \%$ borax additive slightly decreased $\varepsilon^{\prime \prime}$ values at low frequencies, $50 \mathrm{wt} \%$ borax additive enhanced $\varepsilon^{\prime \prime}$ values by $10^{3}$ times. In this context, the maximum dielectric loss has been observed for PPy-50 wt $\%$ borax composite. The PPy-borax composites with the 



Figure 5. (a) $M^{\prime}=f(\omega)$ and (b) $M^{\prime \prime}=f(\omega)$ spectra of pure PPy and PPy-borax composites.

highest dielectric loss may be suitable for decoupling capacitor applications [25].

To decide the polarization type and relaxation mechanism of each samples, the angular frequency dependence of both the real and imaginary component of complex electrical modulus have also been investigated. Complex electrical modulus $M^{*}(\omega)$ is the reciprocal of complex dielectric function $\varepsilon^{*}(\omega)$ :

$$
M^{*}(\omega)=\varepsilon^{*}(\omega)^{-1}=\left[\varepsilon^{\prime}(\omega)-i \varepsilon^{\prime \prime}(\omega)\right]^{-1} .
$$

The angular frequency dependences of the real and imaginary components of the complex electrical modulus for the samples have been shown in figure $5 \mathrm{a}$ and $\mathrm{b}$, respectively.

According to figure $5 \mathrm{a}$, the real part of complex electrical modulus takes values that approach zero at low angular frequencies and increase with increasing angular frequency. This characteristic of $M^{\prime}$ indicates that electrode polarization effect can be ignored [31,32].

Comparison of the imaginary components of $M^{*}(\omega)$ and $\varepsilon^{*}(\omega)$ also gives an information about the conduction process and relaxation mechanism [33]. While pure conduction process manifests itself as a relaxation peak, which is observed only in $M^{\prime \prime}=f(\omega)$, localized dielectric relaxation process identifies with peaks observed in both $M^{\prime \prime}=f(\omega)$ and $\varepsilon^{\prime \prime}=f(\omega)$ spectra [34-36].

As shown in figure $5 \mathrm{~b}$, except for PPy-50 wt $\%$ borax composite, other samples exhibit a relaxation peak. Observation of relaxation peak suggests a transition of the motion of charge carriers from long range to short range with increasing frequency. Moreover, observation of a relaxation peak only in $M^{\prime \prime}=f(\omega)$ for the samples implies that pure conduction process is valid. However, increasing $M^{\prime \prime}$ values above $1 \mathrm{MHz}$ for PPy-50 wt\% borax composite also suggest that a relaxation peak may be observed at higher frequencies. In this context, it may be deduced that borax additive shifts the relaxation frequency to higher frequencies (see the table in figure $5 b$ ) due to the increase in free charge carriers in the material.
Furthermore, since all relaxation peaks have been observed at high frequencies, charge carriers in these materials exhibit short range mobility. The short range mobility of charge carriers in these materials indicates that charge carriers can move in their potential well [37].

\subsection{Complex impedance analysis}

Complex impedance analysis has also been utilized for separating out the grain boundary and grain-electrode effects. In addition, complex impedance analysis also enables calculation of different contributions to the conductivity, namely the bulk, grain boundary and grain-electrode contributions. Towards this purpose, Nyquist plots of the samples given in figure 6 have been analysed.

As shown in figure 6, except for PPy-50 wt $\%$ borax composite, all curves have a tendency to become a semicircle in high-frequency region and a straight line with a small slope in low-frequency region. The centres of the semicircles were also below the real axis, which confirms the non-Debye type of relaxation which had already been determined by nonzero absorption coefficient values obtained by fitting (see table 3). For PPy-50 wt\% borax, it has been observed that the low-frequency curve tends to bend towards the abscissa to form semicircle and has a straight line with a large slope. It has been recognized that a tendency for completing semicircle behaviour observed in figure 6 suggests the presence of both localized and non-localized conduction processes in the materials.

Due to this reason, these Nyquist plots have been fitted for calculating grain and grain boundary parameters, including grain resistance and capacitance $\left(R_{\mathrm{g}}\right.$ and $\left.C_{\mathrm{g}}\right)$, grain relaxation frequency $\left(f_{\mathrm{g}}\right)$ and grain boundary resistance $\left(R_{\mathrm{gb}}\right)$. The fitted Nyquist curves for each sample have been presented in figure 6.

According to the fitted curve in figure 6, a relatively small semicircle drawn at high frequencies indicates the effect of grain and a larger semicircle drawn at low frequencies refers 

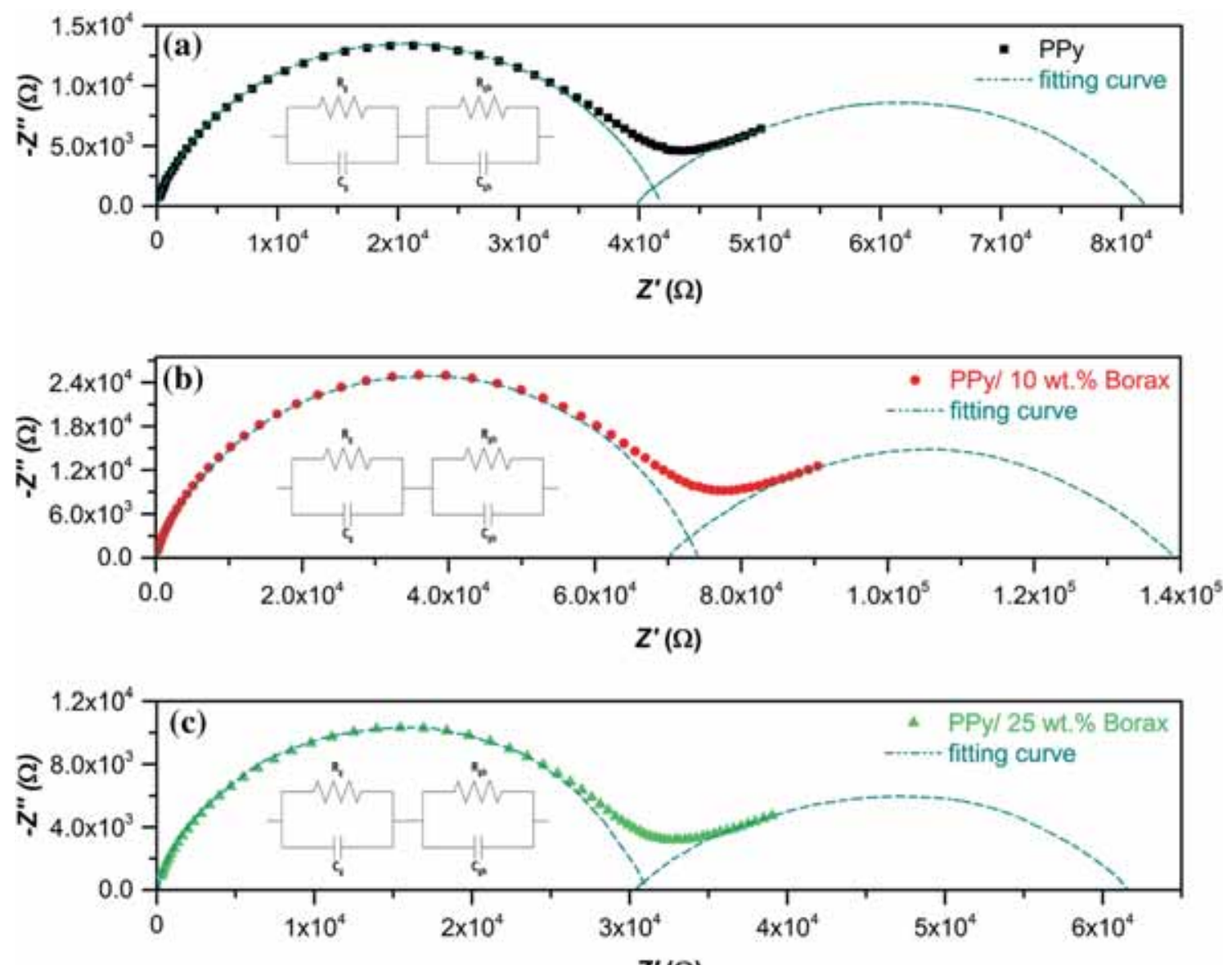

$Z^{\prime}(\Omega)$

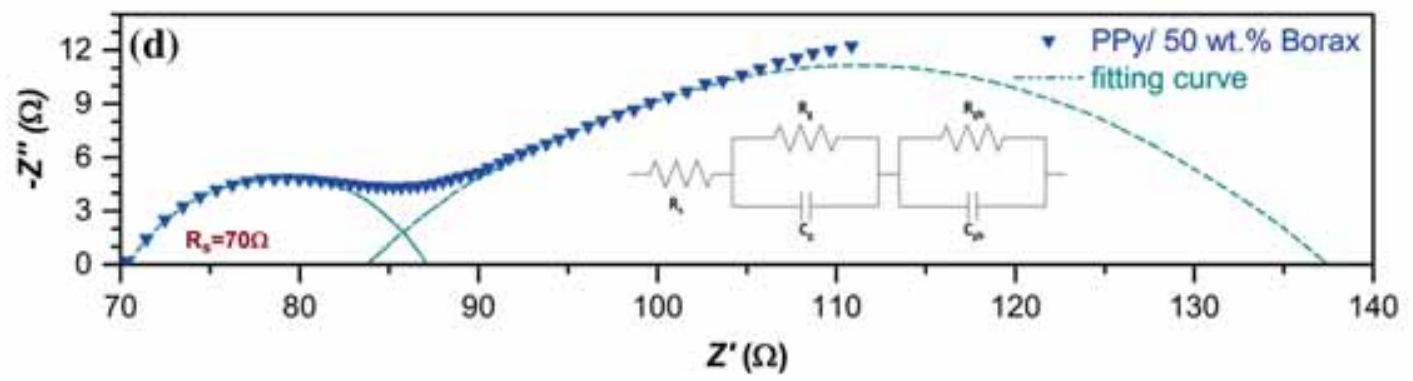

Figure 6. The Nyquist and fitted plots of (a) pure PPy, (b) PPy-10 wt\% borax, (c) PPy-25 wt \% borax and (d) PPy-50 wt\% borax composites.

Table 3. Extracted parameters of the circuit elements of pure PPy and PPy-borax composites.

\begin{tabular}{lrccc}
\hline Sample & $f_{\mathrm{g}}(\mathrm{Hz})$ & $R_{\mathrm{g}}(\Omega)$ & \multicolumn{1}{c}{$R_{\mathrm{gb}}(\Omega)$} & $C_{\mathrm{g}}(\mathrm{F})$ \\
\hline PPy & 70880 & $4.179 \times 10^{4}$ & $8.201 \times 10^{4}$ & $5.372 \times 10^{-11}$ \\
PPy-10 wt\% borax & 59067 & $7.399 \times 10^{4}$ & $13.875 \times 10^{4}$ & $3.641 \times 10^{-11}$ \\
PPy-25 wt\% borax & 146980 & $3.110 \times 10^{4}$ & $6.148 \times 10^{4}$ & $3.481 \times 10^{-11}$ \\
PPy-50 wt\% borax & 365730 & 87.110 & 137.226 & $4.995 \times 10^{-9}$ \\
\hline
\end{tabular}


to the grain boundary effects. The intercepts of the small and large semicircle on $Z^{\prime}$ axis correspond to the resistance $R_{\mathrm{g}}$ and $R_{\mathrm{gb}}$, respectively. The related $C_{\mathrm{g}}$ capacitances have also been calculated by the relation

$$
\omega \tau=2 \pi f_{\mathrm{r}} R C=1,
$$

where $f_{\mathrm{r}}$ is relaxation frequency, at which the maximum $Z^{\prime \prime}$ is achieved, and $\tau$ is relaxation time. The related parameters obtained from Nyquist plots' fitting have been summarized for pure PPy and PPy-borax composites in table 3.

In table 3 , the $C_{\mathrm{g}}$ capacitance values have been determined to be in the order $10^{-11}$ and $10^{-9} \mathrm{~F}$. These values are in good agreement with the grain capacitance values [38]. In addition, the highest and lowest $R_{\mathrm{gb}}$ resistance has been achieved for the composites doped with 10 and $50 \mathrm{wt} \%$ borax, respectively. On the other hand, it is clear that each $R_{\mathrm{g}}$ value is much lower than the related $R_{\mathrm{gb}}$ resistance value. From this point of view, it can be deduced that the semicircle of grain is masked in a limited frequency range. The higher boundary resistance values may be explained by the fact that the atomic arrangement near the grain boundary is disordered, which results in trapped electrons in grain boundaries [38,39].

\section{4 ac Conductivity analysis}

The angular frequency dependence of conductivity, $\sigma_{\mathrm{ac}}$, in solids is generally analysed using Jonscher's power law [40] given in equation (7):

$$
\sigma_{\mathrm{ac}}(\omega)=\sigma(0)+\sigma(\omega)=\sigma_{\mathrm{dc}}+A \omega^{s},
$$

where the first term is frequency-independent conductivity $(\sigma(0))$, which corresponds to direct current $(\mathrm{dc})$ conductivity, and the second term $\left(A \omega^{s}\right)$ is frequency-dependent conductivity. In equation (7), ' $A$ ' is a constant coefficient that defines the strength of polarization, and frequency exponent ' $s$ ' represents the degree of interaction between mobile ions with the lattices around them and generally varies between zero and one $[41,42]$.

The frequency-dependent electrical conductivity, $\sigma_{\mathrm{ac}}$, has been derived from $\varepsilon^{\prime \prime}(\omega)$ data and shown in figure 7 . The frequency exponent ' $s$ ' has been calculated by the slope of $\ln \sigma_{\mathrm{ac}}=f(\ln \omega)$ function of the samples for determining suitable charge transport mechanism for the samples.

It can be clearly seen from the table in figure 7 that the lowfrequency conductivity behaviour of all samples corresponds to 'nearly dc conductivity' due to very low ' $s$ ' values at the vicinity of zero. This tendency continues to high frequencies only for PPy-50 wt\% borax composite. On the other hand, PPy-50 wt\% borax composite has also the highest conductivity values relative to other samples. In this context, observation of high conductivity values together with behaviour that is nearly independent of frequency also suggests that this composite could be utilized as an antistatic

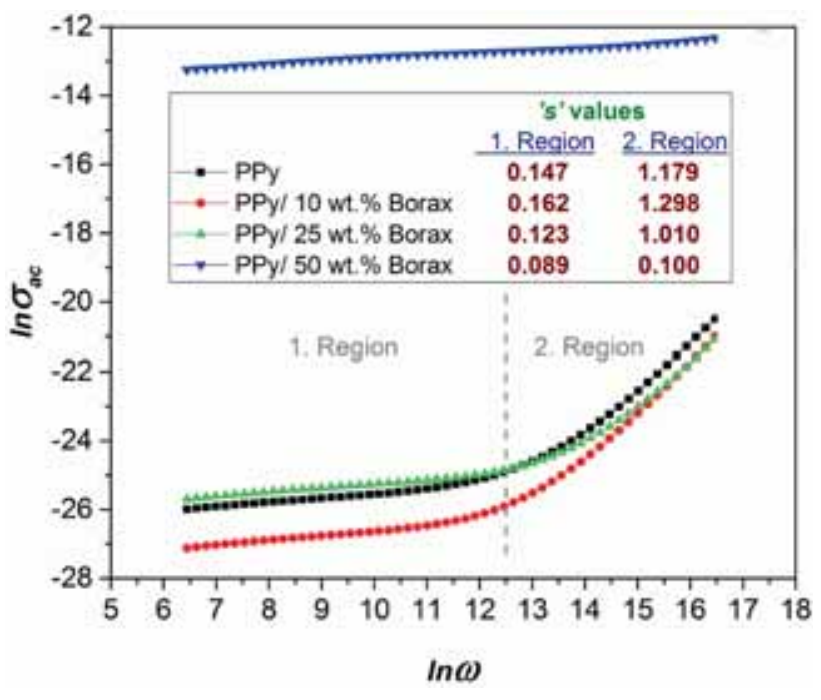

Figure 7. The curves for PPy and PPy-borax composites.

media [25]. Moreover, the other samples, including pure PPy, exhibit ac conductivity in the high-frequency region. Regarding frequency exponent values, while pure PPy and PPy-10 wt\% borax composite showed super-linear power law (SLPL), PPy-25 wt\% borax exhibited a nearly constant loss (NCL) mechanism.

The NCL phenomenon was discovered by Lee et al [43] and is characterized by almost constant $\varepsilon^{\prime \prime}$ over wide ranges of both frequency and temperature. The NCL regime is frequently observed as a universal property of highly disordered materials. According to Ke et al [44], NCL is more likely caused by the vibrational relaxation of the polymer chains. SLPL has also been proposed as a new universal power law for most of the disordered materials by Lukenheimer and Loidl [45].

\section{Conclusions}

In the present study, PPy-borax composites with different borax contents varying from 10 to $50 \mathrm{wt} \%$ have been prepared to obtain materials with different desirable dielectric properties. The structural and morphological properties of the composites have been characterized by FT-IR spectroscopy and scanning electron microscopy, respectively. It has been found that borax additive increases the real part of complex dielectric function of pure PPy in the low-frequency region. This property may make the borax composites suitable for charge storing devices. On the other hand, the imaginary part of complex dielectric function does not exhibit any relaxation peak and is very high in the low-frequency region for the composites. Due to this reason, it has been suggested that the composites with high borax content may also be used in decoupling capacitor applications. The relaxation mechanisms of the pure PPy and PPy-borax composites 
have also been determined as non-Debye type by considering non-zero absorption coefficient values obtained by the fitting of $\varepsilon^{\prime}=f(\omega)$ curves with respect to Cole-Cole equation.

Using the complex plane representation of impedance, Nyquist curves of the samples have also been fitted for determining grain and grain boundary resistance and capacitance values. The related grain and grain boundary capacitance values are in good agreement with the typical grain and grain boundary capacitance values. The Nyquist plots have also confirmed the non-Debye-type relaxation.

The variations of the imaginary component of complex electrical modulus with angular frequency of the samples have been investigated for determining relaxation process. Due to observation of a relaxation peak at high frequencies only for $M^{\prime \prime}=f(\omega)$ curves, the pure conduction process together with short range mobility of charge carriers has been suggested.

The $\ln \sigma_{\mathrm{ac}}=f(\ln \omega)$ curves have showed that PPy-borax composites with the highest borax content may be utilized as an antistatic media due to observation of approximately frequency-independent high conductivity values. In conclusion, it has been revealed that borax, which has been usually used as a low-cost fire retardant in wood industry, has a promising potential for improving the dielectric properties of PPy for electronic applications.

\section{References}

[1] Ballav N and Biswas M 2005 Polym. Int. 54725

[2] Liu R and Liu Z 2009 Chin. Sci. Bull. 542028

[3] Shown I, Ganguly A, Chen L and Chen K 2015 Energy Sci. Eng. 32

[4] Chang K C, Hsu C H, Peng C W, Huang Y Y, Yeh J M, Wan H $\mathrm{P}$ et al 2014 Express Polym. Lett. 8207

[5] Jose J P, Mhetar V, Culligan S and Thomas S 2013 Sci. Adv. Mater. 5385

[6] Kurachi K and Kise 1994 Polym. J. 261325

[7] Wang Y, Sotzing G A and Weiss R A 2008 Chem. Mater. 20 2574

[8] Wanekaya A K, Lei Y, Bekyarova E, Chen W, Haddon R, Mulchandani A et al 2006 Electroanalysis 181047

[9] Cavdar A D, Mengeloğlu F and Karakus K 2015 Measurement 606

[10] Gumus O Y, Unal H I, Erol O and Sari B 2011 Polym. Compos. 32418

[11] Lin H L, Liu Y F, Yu T L, Liu W H and Rwei S P 2005 Polymer 465541

[12] Gao S, Guo J and Nishinari K 2008 Carbohydr. Polym. 72315

[13] Kim S H, Hyun K, Moon T S, Mitsumata T, Hong J S, Ahn K $\mathrm{H}$ et al 2005 Polymers 467156

[14] Basavaraja C, Veeranagouda Y, Lee K, Vishnuvardhan T and Pierson R 2010 J. Polym. Res. 17233
[15] Maity A and Sinha Ray S 2008 Macromol. Rapid Commun. 29 1582

[16] Nicho M and Hu H 2000 Sol. Energy Mater. Sol. Cells 63 423

[17] Gao F, Hou X, Wang A, Chu G, Wu W, Chen J et al 2016 Particuology 2673

[18] Karim M R, Lee C J, Chowdhury A S, Nahar N and Lee M S 2007 Mater. Lett. 611688

[19] Yang C, Liu P, Guo J and Wang Y 2010 Synth. Met. 160592

[20] Heller G 1986 K C Buschbeck and K Niedenzu (eds) Boron and oxygen in: B boron compounds 3rd suppl vol 2 (Berlin: Springer-Verlag) p 166

[21] Gönen M 2009 Nanosized zinc borate production (Izmir: Izmir Institute of Technology)

[22] Koops C 1951 Phys. Rev. 83121

[23] Maxwell J 1873 Electricity and magnetism (London: Oxford University Press)

[24] Wagner K W 1913 Ann. Phys. 40817

[25] Panwar V, Park J O, Park S H, Kumar S and Mehra R M 2010 J. Appl. Polym. Sci. 1151305

[26] Cole K S and Cole R H 1941 J. Chem. Phys. 9341

[27] Haase W and Wrobel S 2003 Relaxation phenomena: liquid crystals, magnetic systems, polymers, high-Tc superconductors, metallic glasses (Berlin: Springer-Verlag)

[28] Meller A 1983 Gmelin handbuch der anorganische chemie, boron compounds (Berlin: Springer-Verlag)

[29] Vassilikou-Dova A and Kalogeras I M 2009 in: J D Menczel and R B Prime (eds) Thermal analysis of polymers: fundamentals and applications (New Jersey: Wiley) p 497

[30] Adam N, Uğur A L, Altındal A and Erdoğmuş A 2014 Polyhedron 6832

[31] El-Gamal S, Ismail A M and El-Mallaway R 2015 J. Mater. Sci.: Mater. Electron 267544

[32] Qureshi A, Singhi N L, Shah S, Singh F and Avasthi D K 2008 J. Macromol. Sci. 45265

[33] Liu J, Duan C G, Yin W G, Mei W N, Smith R W and Hardy J R 2003 J. Chem. Phys. 1192812

[34] Angell C A 1990 Chem. Rev. 90523

[35] Hodge I M, Ingram M D and West A R 1976 J. Electroanal. Chem. Interfacial Electrochem. 74125

[36] Gerhardt R 1994 J. Phys. Chem. Solids 551491

[37] Prakash T, Prasad K P, Kavitha R, Ramasamy S and Murty B S 2007 J. Appl. Phys. 102104104

[38] Mahamoud H, Louati B, Hlel F and Guidara K 2011 J. Alloys Compd. $\mathbf{5 0 9} 6083$

[39] Kaushal A, Olhero S M, Singh B, Fagg D P, Bdikin I and Ferreira J M F 2014 Ceram. Int. 4010593

[40] Jonscher A K. 1983 Dielectric relaxation in solids (London: Chelsea Dielectrics Press)

[41] Jonscher A K 1999 J. Phys. D: Appl. Phys. 32 R57

[42] Rao K S, Krishna P M, Prasad D M, Latha T S and Satyanarayana C 2008 Indian J. Eng. Mater. Sci. 15215

[43] Lee W K, Liu J F and Nowick A S 1991 Phys. Rev. Lett. 67 1559

[44] Ke S, Huang H, Ren L and Wang Y 2009 J. Appl. Phys. 105 096103

[45] Lunkenheimer P and Loidl A 2003 Phys. Rev. Lett. 91207601 